\title{
Modeling autoregulation in three-dimensional simulations of retinal hemodynamics
}

Matteo Aletti, Jean-Frédéric Gerbeau, Damiano Lombardi

Inria Paris \& Sorbonne Universités, UPMC Univ, Paris 6, France

\section{Abstract}

Purpose: Autoregulation is a mechanism necessary to maintain an approximately constant blood flow rate in the microcirculation when acute changes in systemic pressure occur. Failure of autoregulation in the retina has been associated with various diseases, including glaucoma. In this work, we propose an initial attempt to model autoregulation in a 3D network of retinal arteries.

Methods: The blood flow is modeled with the time-dependent Stokes equations. The arterial wall model includes the endothelium and the smooth muscle fibers. Various simplifying assumptions lead to a fluid-structure model where the structural part appears as a boundary condition for the fluid. The numerical simulations are performed on a patient-specific network of 25 segments of retinal arteries located in the inferior temporal quadrant.

Results: The simulations performed on the patient-specific artertial network have provided velocities which are in good agreement with published experimental data. In addition, the model allowed to reproduce flow rate-pressure curves which are comparable with experimental data or results obtained with 0D models. In particular, a characteristic plateau of the flow rate has been found for pressures ranging from 40 to $60 \mathrm{mmHg}$.

Conclusion: This work proposes the first 3D simulation of blood flow in a real network of retinal arteries and it also incorporates an autoregulation mechanism. This can be viewed as a first step towards a more complete 3D model of the hemodynamic of the eye.

Keywords: retina; autoregulation; 3D hemodynamics

Correspondence: Matteo Aletti, Inria Paris \& Sorbonne Universités UPMC Univ Paris 6 , France

E-mail: matteo.aletti@inria.fr 


\section{Introduction}

Retinal hemodynamics is strongly influenced by vascular autoregulation. This mechanism is necessary to maintain an approximately constant flow rate in the microcirculation when acute changes in the pressure occur, and it is present in various tissues and organs. ${ }^{1}$ Failure or impairment of autoregulation in the retina has been associated with various diseases, for instance, diabetic retinopathy and glaucoma. ${ }^{2,3,4}$

In the retina, this phenomenon has been studied both in animals ${ }^{5}$ and in humans. ${ }^{6,7,8,9,10,11}$ Retinal vessels contract or relax in response to a change in the perfusion pressure or to a specific metabolic need. However, the mechanisms underlying the metabolic pathways that trigger vessel contraction or relaxation are still under investigation.

Autoregulation has been modeled on OD networks of arterioles ${ }^{12}$ and also specifically in the retina. ${ }^{13}$ The present work is an initial attempt to address this phenomenon with 3D patient-specific networks, focusing on the mechanical aspects.

This work is not motivated by any specific clinical application. Its goal is to propose a first step toward a 3D model of the hemodynamics of the eye. Even if existing OD models can provide valuable information, we believe that 3D models can be useful to better understand the complex mechanical interactions which occur within the eye. With modern segmentation tools, patient-specific vasculature can be automatically reconstructed from retinal fundus images. With 3D models, it will be possible to use these rich data to address new issues where geometry plays an important role. For instance, it could be interesting to investigate the blood flow when a dysfunction occurs in a very localized part of the retina. Venular-arteriolar communication ${ }^{14}$ gives another example where a precise representation of the geometry would also be useful. Nowadays, it is even possible to acquire video of the retinal vasculature showing the pulsatility of the arterial wall. A data assimilation procedure in a 3D fluid-structure model could allow us to estimate the local mechanical properties of the vessel, as was done for the aorta. ${ }^{15,16}$

The numerical simulation of autoregulation in $3 \mathrm{D}$ requires a system of equations that model the mechanical interaction between the blood and the arterial wall. Various approaches have been proposed in the literature to address this problem in large arteries. The most complete models are based on the nonlinear elastodynamics equation coupled with the Navier-Stokes equations set in a moving domain. We refer, for example, to the monograph by Fernández and Gerbeau ${ }^{17}$ or to one of the many others available ${ }^{18,19,20,21}$, to name but a few. These models are very demanding from a computational viewpoint and are valid for large displacements, which is not always necessary, especially in small arteries. Less expensive approaches have been proposed, where the arterial wall equation is drastically simplified. ${ }^{22,23,24}$ The model used in the present work adopts this latter approach, but it introduces new features, such as active fibers, which are useful when addressing the autoregulation problem. In the study by Colciago et al. ${ }^{25}$ the authors observed that using simplified models 
on image-based geometry might cause numerical instabilities, due to inaccurate approximations of the normals and the principal curvatures of the surface. A side effect of including fibers in this model is the improvement of the numerical stability, owing to a Laplace operator added to the structure equation. This model has been recently proposed in the study by Aletti et al., ${ }^{26}$ where a complete derivation and the numerical schemes can be found. The novelty of the present work lies in its modeling the autoregulation mechanism and the numerical simulation of this phenomenon in patient-specific retinal arteries. The numerical tests are carried out on a portion of the retinal vasculature consisting of 25 arterial segments located in the inferior-temporal quadrant and reconstructed from a retinal fundus image.

The structure of the work is as follows: in Section 2, the fluid-structure framework is introduced; Section 3 addresses the structural model, with special emphasis on the fibers. Section 4 deals with the autoregulation mechanism and Section 5 presents the numerical results. Section 6 presents some limitations of the study and the conclusion.

\section{Fluid-structure coupling: main modeling assumptions}

The first modeling assumption is to neglect the convective terms in the fluid momentum equation. This approximation is justified since, by considering a maximum vessel diameter $D=200 \mu \mathrm{m}$, a velocity $v=5 \mathrm{~cm} / \mathrm{s}$ and a kinematic viscosity $\nu=0.04 \mathrm{~cm}^{2} / \mathrm{s}$, we obtain a Reynolds number of 2.5 . The second main assumption is to suppose that the blood behaves as an homogeneous Newtonian fluid, which is questionable since microvessels are considered. ${ }^{27,28}$ We make this hypothesis for the sake of simplicity because it is assumed not to affect too much the autoregulation, which is the mechanism this work focuses on.

The domain $\Omega_{t}$ in which the fluid flows, is in general, time-dependent, since the wall is an elastic structure in interaction with the fluid. The boundary $\partial \Omega_{t}$ is subdivided into two subsets: $\Gamma_{t}$, which is the interface between the fluid and the structure, and $\Sigma_{t}$, representing the artificial boundaries of the domain where inlet and outlet boundary conditions are enforced. Considering the small displacements of the retinal arteries wall, the domain $\Omega_{t}$ is considered to have a fixed reference configuration denoted by $\Omega$. A schematic representation of this setting is given in Fig. 1. This approximation considerably reduces the computational cost. The fluid equations are therefore:

$$
\left\{\begin{array}{l}
\rho^{f} \partial_{t} \boldsymbol{u}=\nabla \cdot \boldsymbol{\sigma}^{f} \quad \text { in } \Omega, \\
\nabla \cdot \boldsymbol{u}=0 \text { in } \Omega,
\end{array}\right.
$$

where $\boldsymbol{u}$ is the velocity, $\rho^{f}$ is the fluid density and $\boldsymbol{\sigma}^{f}=\mu^{f}\left(\nabla \boldsymbol{u}+(\nabla \boldsymbol{u})^{T}\right)-p \boldsymbol{I}$ is the fluid stress tensor, where $\mu^{f}$ is the dynamic viscosity and $p$ is the pressure.

The velocity on the fixed fluid-structure interface $\Gamma$ is obtained by a Taylor expansion. This approach is known as a 'transpiration condition'. In the literature, this is 


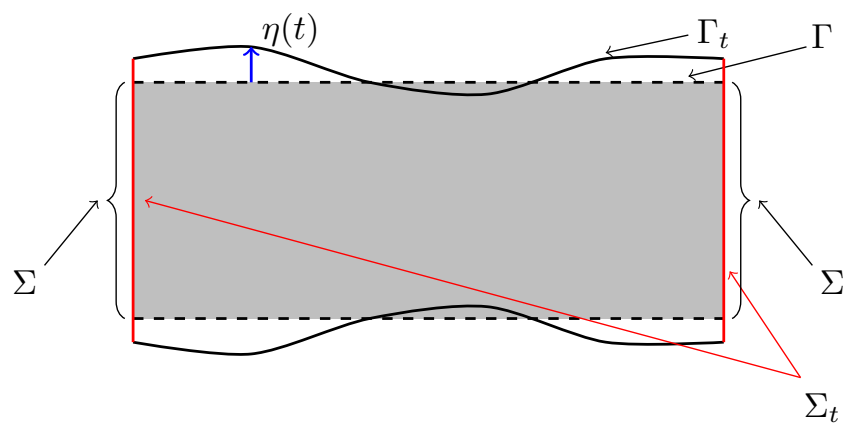

Figure 1: The fluid domain $\Omega_{t}$ is within the two curved lines, however the equations are solved on the fixed computational domain $\Omega$ depicted in gray. The displacement field $\eta$, which depends on time, maps $\Gamma$ into $\Gamma_{t}$.

usually a zero-th order expansion. Here, a first order transpiration condition is adopted in order to compute the variation of the flow induced by the wall dynamics, which is important in order to model autoregulation. The main geometrical assumption is that the normal to the structure $\boldsymbol{n}$ is constant in time. Moreover, the kinematics of the vessel wall is assumed to be, at each time, parallel to the normal. With $\boldsymbol{\eta}$ denoting the displacement of the wall, the following holds:

$$
\boldsymbol{\eta}=\eta \boldsymbol{n}, \forall t .
$$

Two conditions have to be satisfied on the fluid-structure interface $\Gamma_{t}$ : the continuity of the velocity and the continuity of the stress. Since the structure displacement is assumed to be parallel to the normal direction, the equations for the continuity of the velocity are, for all $x \in \Gamma,\left.\boldsymbol{u}(\boldsymbol{I}-\boldsymbol{n} \otimes \boldsymbol{n})\right|_{\boldsymbol{x}+\eta(\boldsymbol{x}) \boldsymbol{n}(\boldsymbol{x})}=\mathbf{0}$ and $\boldsymbol{u}$. $\left.\boldsymbol{n}\right|_{\boldsymbol{x}+\eta(\boldsymbol{x}) \boldsymbol{n}(\boldsymbol{x})}=\partial_{t} \eta$. The balance of the normal component of the normal stress gives $\left.\boldsymbol{\sigma}_{\boldsymbol{n} \boldsymbol{n}}^{f}\right|_{\boldsymbol{x}+\eta(\boldsymbol{x}) \boldsymbol{n}(\boldsymbol{x})}=-f^{s}-p_{i o p}$, where $p_{i o p}$ denotes the external pressure acting on the structure, in this case the intra ocular pressure, and $f^{s}$ represents the stress coming from the structure.

The simplifying assumptions on the structure dynamics, which will be detailed in the following section, allow us to treat the structure equations as a boundary condition for the fluid problem.

The equations for the coupled system are written in weak form, on a fixed domain. Let $\boldsymbol{v}, q, \chi, \boldsymbol{w}$ be test functions defined in suitable functional spaces according to the boundary conditions of the problem. In particular let $\boldsymbol{u}(t)$ and $\boldsymbol{v} \in \mathbf{V}$, let $p(t)$ and $q \in M$, where $V=\mathbf{H}^{2}(\Omega)$ and $M=H^{1}(\Omega)$. Then: 


$$
\begin{cases}\left\langle\partial_{t} \boldsymbol{u}, \boldsymbol{v}\right\rangle_{\Omega}+a(\boldsymbol{u}, \boldsymbol{v})+b(p, \boldsymbol{v})=0 & \text { in } \Omega, t>0 \\ \langle\nabla \cdot \boldsymbol{u}, q\rangle_{\Omega}=0 & \text { in } \Omega, t>0 \\ \rho_{s} h_{s}\left\langle\partial_{t t}^{2} \eta, \chi\right\rangle_{\Gamma}+\Psi^{s}(\eta, \chi)+\left\langle p_{i o p}, \chi\right\rangle_{\Gamma}=\langle p+\eta \nabla p \cdot \boldsymbol{n}, \chi\rangle_{\Gamma} & \text { on } \Gamma \\ \left\langle\partial_{t} \eta, \chi\right\rangle_{\Gamma}=\langle\boldsymbol{u} \cdot \boldsymbol{n}+\eta \nabla \boldsymbol{u n} \cdot \boldsymbol{n}, \chi\rangle_{\Gamma} & \text { on } \Gamma \\ \langle(\boldsymbol{I}-\boldsymbol{n} \otimes \boldsymbol{n})(\boldsymbol{u}+\eta \nabla \boldsymbol{u n}), \boldsymbol{w}\rangle_{\Gamma}=0 & \text { on } \Gamma .\end{cases}
$$

The forms $a, b$ read:

$$
\begin{array}{lll}
a: \boldsymbol{V} \times \boldsymbol{V} \rightarrow \mathbb{R}, & a(\boldsymbol{u}, \boldsymbol{v})=\nu^{f}\left\langle\nabla \boldsymbol{u}+\nabla \boldsymbol{u}^{T}, \nabla \boldsymbol{v}\right\rangle_{\Omega} & \forall(\boldsymbol{u}, \boldsymbol{v}) \in \boldsymbol{V} \times \boldsymbol{V} \\
b: M \times \boldsymbol{V} \rightarrow \mathbb{R}, & b(p, \boldsymbol{v})=-\langle p, \nabla \cdot \boldsymbol{v}\rangle_{\Omega} & \forall(p, \boldsymbol{v}) \in M \times \boldsymbol{V} .
\end{array}
$$

where $\langle\cdot, \cdot\rangle_{\Omega}$ and $\langle\cdot, \cdot\rangle_{\Gamma}$ denote the standard scalar product in $L^{2}(\Omega)$ and in $L^{2}(\Gamma)$, respectively and $\nu^{f}$ is the kinematic viscosity. It should be noted that in this framework the structure dynamics is embedded as a boundary condition of the fluid problem. The system in Eq.(3) is discretised by means of finite elements (P1-P1, with a SUPG stabilisation) and by a mixed semi-implicit scheme in time. All the details of the implementation of this approach are provided in the study by Aletti et al. ${ }^{26}$

Remark 1. Numerically, the tangential velocity sometimes exhibits oscillations on the fluid-structure interface, especially on complex geometries. The reason for these oscillations is not completely understood. They might be due to the approximation of the normals and the curvatures, as already noted by Colciago et al. ${ }^{25}$, or to the first order transpiration terms adopted in our approach. We observed that this problem can be alleviated by the following consistent stabilization term:

$$
\langle(\boldsymbol{I}-\boldsymbol{n} \otimes \boldsymbol{n})(\boldsymbol{u}+\eta \nabla \boldsymbol{u n}), \boldsymbol{w}\rangle_{\Gamma}=-\beta\langle h \nabla(\boldsymbol{I}-\boldsymbol{n} \otimes \boldsymbol{n}) \boldsymbol{u}, \nabla \boldsymbol{w}\rangle_{\Gamma} \text { on } \Gamma,
$$

where $\beta \geq 0$ is the stabilization coefficient and $h$ is the surface element size.

\section{Modeling the vessel wall dynamics}

Retinal arteriolar structure consists of a thin layer of endothelium layer and a layer of smooth muscle cells which is more developed with respect to vessels of the same size in other organs. ${ }^{28}$ From a modeling perspective, the wall is considered as an elastic shell, so as to render the behavior of the endothelium, and several fiber layers to model the smooth muscles.

This section is organised as follows. After introducing the notation, the model of the structure is presented in its general form, as it appears in system (3). The model for the endothelium is described, followed by a presentation of the fiber layer. First a derivation of a constitutive law for the smooth muscle fibers is presented. This constitutive law is then used to close the kinematical and mechanical description of the fiber layer surrounding the endothelium. 


\subsection{Notation}

Let $\Gamma$ be the reference position of the vessel wall, i.e., the position at which it is in a normal state of equilibrium, without external influences. The geometrical configuration of $\Gamma$ is described by a regular map $\Phi$ such that $\boldsymbol{\xi} \in \omega \subseteq \mathbb{R}^{2} \mapsto \boldsymbol{x}=\Phi(\boldsymbol{\xi}) \in \Gamma$. Let $\boldsymbol{A}$ be the first fundamental form and $\boldsymbol{B}$ the second fundamental form associated with the reference configuration $\Gamma$. Let $\boldsymbol{S}=\boldsymbol{A}^{-1} \boldsymbol{B}$ be the representation of the shape operator. The eigenvalues of the shape operator are the principal curvatures of the surface $\Gamma$, the mean curvature being the average of the principal curvatures and the Gaussian curvature being their product. The surface parametrization is denoted by Greek letters and the curvilinear coordinates domain is denoted by $\omega \subseteq \mathbb{R}^{2}$.

\subsection{Equations for the structure dynamics}

The equations for the structure dynamics, appearing as a boundary condition of the system (3), are obtained by adding the inertia terms to the elastic energy of the structure. In particular, the dynamics equations in weak form can be written as:

$$
\int_{\omega} \rho^{s} h^{s}\left(\partial_{t t}^{2} \eta\right) \chi \sqrt{a} d \boldsymbol{\xi}+\Psi^{s}(\eta, \chi)=0,
$$

where the thickness of the structure is denoted by $h^{s}$ and its density by $\rho^{s}$. The form $\Psi^{s}$ describes the behavior of the structure. It is considered as the sum of several contributions:

$$
\Psi^{s}=\Psi^{\kappa}+\Psi^{\mathbf{w}}+\Psi^{\mathbf{v}}
$$

where $\Psi^{\kappa}$, defined in Eq.(14), represents the contribution of the endothelium and $\Psi^{\mathbf{w}}, \Psi^{\mathbf{v}}$, defined in Eq.(35), represent the contribution of the fibers aligned in the directions $\mathrm{w}$ and $\mathrm{v}$ respectively.

Each of these terms is analyzed in detail in the following part of this section. In general, all the weak forms are derived as follows: given an elastic model and the corresponding energy, the equilibrium configuration for the structure can be seen as the stationary point of the energy functional:

$$
\Psi(\eta, \chi):=\left\langle\delta_{\eta} \psi(\eta), \chi\right\rangle_{\Gamma}=0,
$$

where $\delta_{\eta}$ denotes the Frechet derivative with respect to $\eta$ and $\psi$ is the elastic energy.

\subsection{The endothelium layer}

A nonlinear Koiter shell model is adopted to describe the endothelium dynamics. The equations and a detailed mathematical derivation are presented in the work by Ciarlet. ${ }^{29}$ The choice of this nonlinear model, rather than the simpler linear version, is motivated by the consistency with the fiber layer description. In particular, when the fiber kinematics (see Eq.(31)) is described, some nonlinear contributions arise. The 
terms appearing in the Koiter model that have the same order with respect to the displacement field $\eta$ have thus to be kept. The displacement field, as pointed out in Eq.(2), is parallel to the normal to the reference configuration.

The simplifying hypotheses from a mechanical point of view are the following:

- the bending terms are negligible;

- the material is linear, isotropic and homogeneous.

The equilibrium configuration is the stationary point of the energy functional: ${ }^{29}$

$$
\psi^{\kappa}(\eta)=\frac{1}{2} \int_{\omega} \mathcal{E}^{\alpha \beta \sigma \tau} g_{\sigma \tau}(\eta) g_{\alpha \beta}(\eta) h_{\kappa} \sqrt{a} d \boldsymbol{\xi}-\int_{\omega} \boldsymbol{f} \cdot \eta h^{\kappa} \sqrt{a} d \boldsymbol{\xi}
$$

where $\sqrt{a}=\sqrt{\operatorname{det}(A)}, h^{\kappa}$ is the shell thickness and $\boldsymbol{f}$ are the external forces, $g(\eta)$ is the change of metric tensor. The properties of the material are contained in the elastic tensor $\mathcal{E}$, whose contravariant components read:

$$
\mathcal{E}^{\alpha \beta \sigma \tau}=\frac{4 \lambda^{s} \mu^{s}}{\lambda^{s}+2 \mu^{s}} A^{\alpha \beta} A^{\sigma \tau}+2 \mu^{s} A^{\alpha \sigma} A^{\beta \tau}+2 \mu^{s} A^{\alpha \tau} A^{\beta \sigma},
$$

where $\lambda^{s}, \mu^{s}$ are the Lamé coefficients of the structure.

By exploiting the hypothesis of normal displacement (see Eq.(2)), the expression for the change of metric tensor becomes:

$$
g_{\alpha \beta}=-B_{\alpha \beta} \eta+\frac{1}{2} A^{\sigma \tau} B_{\sigma \alpha} B_{\tau \beta} \eta^{2}+\frac{1}{2} \partial_{\alpha} \eta \partial_{\beta} \eta,
$$

where the derivative with respect to the Greek letters denotes the derivation with respect to the surface parametrization.

The form $\Psi^{\kappa}$ describing the equilibrium of the nonlinear Koiter shell model under the assumptions made reads:

$$
\begin{array}{r}
\Psi^{\kappa}(\eta, \chi):=\frac{2 E}{1-\nu^{2}} \int_{\omega}\left(c_{1} \eta-3 c_{2} \eta^{2}+2 c_{3} \eta^{3}\right) \chi-2 \nabla \chi^{T}\left(C_{1} \eta+C_{2} \eta^{2}\right) \nabla \eta+ \\
-\nabla^{T} \eta\left[\left(C_{1}+2 C_{2} \eta\right) \chi\right] \nabla \eta+\frac{1}{2}\left(\nabla \eta^{T} A^{-1} \nabla \eta\right) \nabla^{T} \chi A^{-1} \nabla \eta h^{\kappa} \sqrt{a} d \boldsymbol{\xi}- \\
\int_{\omega} \boldsymbol{f}_{n} \cdot \chi h^{\kappa} \sqrt{a} d \boldsymbol{\xi}
\end{array}
$$

where $E$ is the Young modulus of the material, $\nu$ the Poisson coefficient, the constant tensors $\left(C_{j}\right)$ and the coefficients $\left(c_{k}\right)$ are expressed as functions of the mean 
and Gaussian curvatures (respectively $\rho_{1}$ and $\rho_{2}$ ) and the Poisson ratio as follows:

$$
\begin{array}{r}
c_{1}=4 \rho_{1}^{2}-2(1-\nu) \rho_{2}, \\
c_{2}=4 \rho_{1}^{3}+(\nu-3) \rho_{1} \rho_{2}, \\
c_{3}=4 \rho_{1}^{4}-4 \rho_{1}^{2} \rho_{2}+\frac{1}{2}(1+\nu) \rho_{2}^{2}, \\
C_{1}=\left[\nu \rho_{1} I+\frac{1}{2}(1-\nu) S\right] A^{-1}, \\
C_{2}=\left[\nu \rho_{1}^{2} I+\frac{1}{2}(1-\nu) S^{2}\right] A^{-1} .
\end{array}
$$

Notice that, at the first order, the endothelium behaves pointwise as a spring with constant $c_{1}$. However the overall behavior can be roughly considered as the sum of two contributions: a nonlinear spring and a nonlinear membrane. More details about this derivation can be found in the study by Aletti et al. ${ }^{26}$

\subsection{The smooth muscle cells model}

In this section, a model describing the behavior of the smooth muscle cells (SMC) is investigated. The resulting model is a 1D idealisation of the SMC muscle fibers. The equations derived in the present section are used as a constitutive law to close the model for the fiber layers.

The layer of SMCs, which in large vessels is also responsible for adaptive changes in the stiffness, has the ability to contract or relax following electrochemical stimuli in order to regulate the blood flow (see, e.g., studies by Murtada et al. and Milnor et al. ${ }^{30,31}$ )

There is no autonomic innervation in the retinal vasculature. ${ }^{32}$ This implies that the regulation is carried out by mechanisms that take place locally in the eye. ${ }^{33}$ The contraction of the smooth muscle cells is controlled by the concentration of calcium ions, which trigger the phosphorylation of myosin light chains. ${ }^{1}$ The different chemical pathways are not investigated in the present work and we make the simplifying assumption that the concentration of calcium ions depends only on changes of the pressure. However, if more sophisticated models are available they can be included to relate the concentration of calcium ions to the other mechanisms.

A chemical state model of the smooth muscle cells has been proposed by Hai and Murphy ${ }^{34}$. This model takes four chemical species into account: myosin $(M)$, phosphorilated myosin $\left(M_{p}\right)$, phosphorilated actin myosin cross-bridge $\left(A M_{p}\right)$ and unphosphorilated actin myosin cross-bridge $(A M)$. It describes the evolution of the concentrations $\left(\alpha_{M}, \alpha_{M_{p}}, \alpha_{A M}, \alpha_{A M_{p}}\right)$ of those species with a linear system of differential equations $\dot{\boldsymbol{\alpha}}=\boldsymbol{K} \boldsymbol{\alpha}$ under the constraint $\alpha_{M}+\alpha_{M_{p}}+\alpha_{A M}+\alpha_{A M_{p}}=1$, where $\boldsymbol{K}$ depends on the concentration of calcium ions.

The chemical model by Hai and Murphy ${ }^{34}$ was used by Yang et al. ${ }^{35,36}$ to describe the myogenic response. More recently, the approach by Yang et al. has been extended 
to a continuum mechanics framework. ${ }^{37,30}$ Such models have also been coupled with a membrane model to compute the concentration of calcium ions as a response to external stimuli. ${ }^{38}$ The overall mechanism is reproduced in the following way: an external stimulus causes a change in calcium concentration which alters the chemical state of the SMC. Once the chemical state is known, it is possible to compute the active component of the forces in the cell and thus its mechanical behavior.

In what follows, we refer to the model of Yang et al. ${ }^{35,36}$ and propose a further simplification in order to derive a constitutive equation for a 1D fiber. In the model presented inthe first study by Yang et al., ${ }^{35}$ the mechanics of a single SMC is described by two elements in parallel: a spring characterized by an exponential force-length relationship (to describe the passive structural behavior of the overall cell) and an active element for the cross-bridges. The total force is given by

$$
F=F_{\text {cell }}+F_{c b}
$$

where $c b$ stands for cross-bridges. The active element is itself made of three elements in series which model the active force of the actin-myosin cross-bridges, their passive elasticity properties and the viscous effects, respectively. The total length of the active element $L_{c b}$ can be expressed as the sum of the length of these three components:

$$
L_{c b}=L_{c b, a}+L_{c b, e l}+L_{c b, v i s c},
$$

where $a, e l$, visc stand for active, elastic and viscous, respectively. The length of the cell and of the active element representing the cross-bridges are the same and they are equal to the total length $L$.

$$
L=L_{\text {cell }}=L_{c b}
$$

The system of equations is closed by observing that the elements in series have the the same force:

$$
F_{c b, a}=F_{c b, e l}=F_{c b, v i s c} .
$$

Finally, considering the constitutive laws of the three components of the active element, it is possible to solve the system and obtain the total force. Such force depends on the total length of the cell, on its time derivative and also on the cell chemical state described by the proportion of phosphorilated and dephosphorilated actin-myosin:

$$
F=F\left(L, \frac{d L}{d t}, \alpha_{A M}, \alpha_{A M_{p}}\right) .
$$

This expression can be simplified if the visco-elastic effects are neglible compared to the elastic effects. In such a case the total force is a function of only the total length and the concentration of phosphorilated actin-myosin:

$$
F=F\left(L, \alpha_{A M_{p}}\right) .
$$




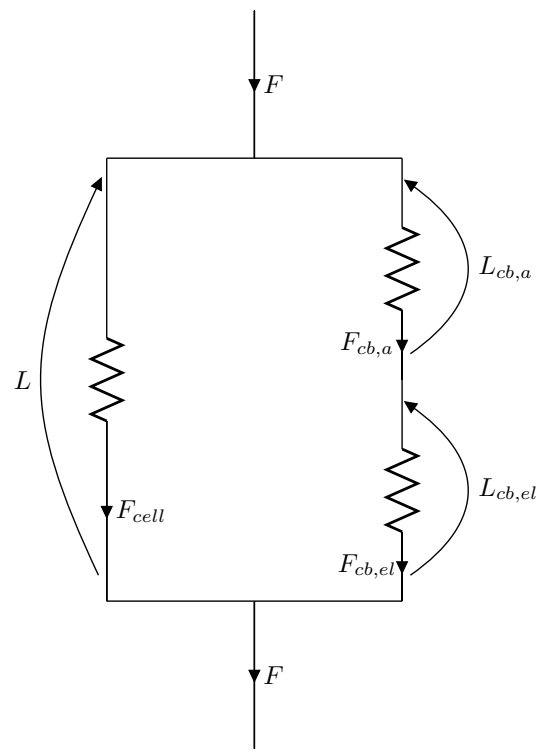

Figure 2: Scheme of the equivalent circuit describing the model by Yang et al. ${ }^{35,36}$ when viscous effects are neglected.

Its expression can be obtained by solving the following system whose equivalent circuit is depicted in Fig.2:

$$
\begin{cases}F_{c e l l} & =k_{c e l l}\left(e^{\alpha_{c e l l}\left(\frac{L}{L_{0}}-1\right)}-1\right) \\ F_{c b, a} & =f_{A M_{p}} \alpha_{A M_{p}} e^{-b\left(\frac{L_{c b, a}}{L_{o p t}}-1\right)^{2}} \\ F_{c b, e l} & =k_{e l}\left(e^{\alpha_{e l}\left(\frac{L_{c b, e l}}{L_{c b, e l, 0}}-1\right)}-1\right) \\ F_{c b, a} & =F_{c b, e l} \\ F & =F_{c e l l}+F_{c b, a} \\ L & =L_{c b, a}+L_{c b, e l},\end{cases}
$$

where the first three equations represent the constitutive law of the cell, the active part of the cross-bridges and their elastic part. Therefore, the parameters $k_{\text {cell }}, \alpha_{\text {cell }}$, $L_{0}, f_{A M_{p}}, b, L_{o p t}, k_{e l}, \alpha_{e l}$ and $L_{c b, e l, 0}$ describe the structural properties of the SMC. The last three equations are obtained by combining equations (20),(21),(22) and (23).

Since it is not possible to obtain a closed-form solution for system (26), we make an approximation to obtain an affine stress-strain relationship. Eq.(25) is linearized with respect to the reference configuration $L_{r e f}$ :

$$
F \sim F_{0}\left(\alpha_{A M_{p}}\right)+F_{1}\left(\alpha_{A M_{p}}\right)\left(L-L_{r e f}\right),
$$




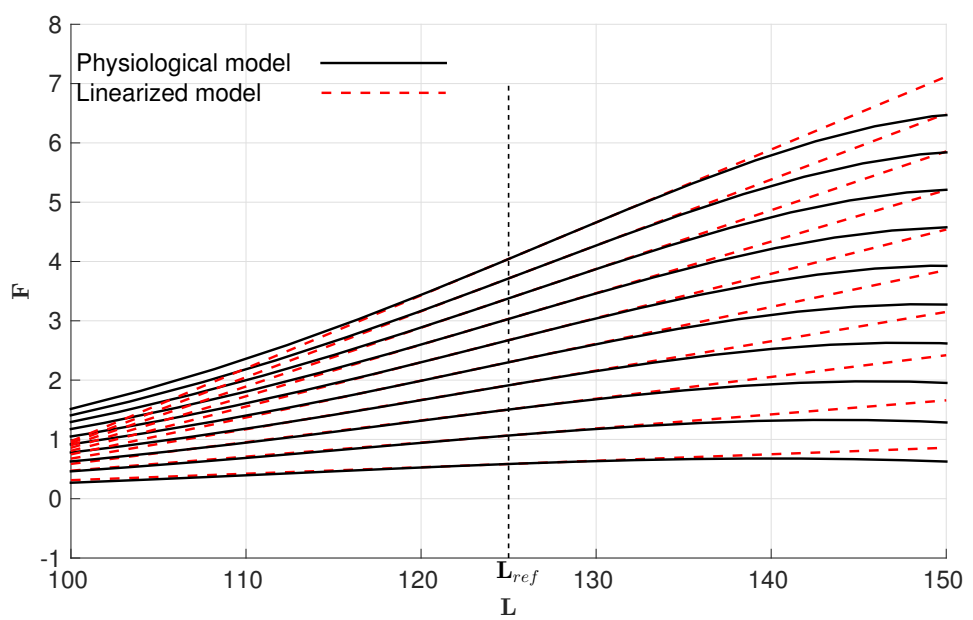

Figure 3: Force-length relationship from system (26), coefficients taken from Yang et al. (2003). Numerical approximation (black) compared with its approximation (dashed red) linearized with respect to the $L_{r e f}$.

where the analytical expressions for $F_{0}$ and $F_{1}$ are known and are derived through a Taylor expansion. In order to corroborate the final result, the physiological model in system (26) is compared with its linearized version for different values of $\alpha_{A M_{p}}$. The result is presented in Fig.3, which shows that a simple affine constitutive law is indeed valid for an approximated description of the SMC fibers for the retina in physiological regimes.

The constitutive law adopted, which is the simplest law that approximately describes the behaviour of the SMCs is:

$$
\sigma_{1 D}=k_{0}+k_{1} \varepsilon_{1 D}
$$

where $\sigma_{1 D}$ is the elastic stress, $k_{0}$ is the pre-stress of the fiber, $k_{1}$ is the elastic modulus, $\varepsilon_{1 D}$ is the fiber deformation.

Equation (27) can finally be used to identify the parameters $k_{0}, k_{1}$, after a rescaling. The SMCs are assumed to have a cylindrical shape with a radius $r_{s m c}$. The force is divided by $\pi r_{s m c}^{2}$ in order to obtain the stress. The result reads:

$$
\begin{aligned}
& k_{0}\left(\alpha_{A M_{p}}\right)=\frac{1}{\pi r_{s m c}^{2}} F_{0}\left(\alpha_{A M_{p}}\right), \\
& k_{1}\left(\alpha_{A M_{p}}\right)=\frac{1}{\pi r_{s m c}^{2}} F_{1}\left(\alpha_{A M_{p}}\right),
\end{aligned}
$$

In practice, it is difficult to have access to the value of $\alpha_{A M_{p}}$, which depends on many 
factors. This is why in the following section, the functions $k_{0}$ and $k_{1}$ will be simply assumed to depend on an 'activation parameter' $\zeta$ :

$$
\sigma_{1 D}=k_{0}(\zeta)+k_{1}(\zeta) \varepsilon_{1 D}
$$

\subsection{The fiber layer}

In this section, the equations describing the dynamics of an SMC fiber layer are detailed. The main hypotheses are the following:

- the fibers are perfectly attached to the shell;

- the fibers are characterized by an affine stress-strain constitutive law.

The kinematic hypothesis implies that the deformation of the fibers equals the deformation of the underlying shell structure in the direction of the fibers.

Let $\mathbf{w} \in \mathbb{T}_{\boldsymbol{x}}(\Gamma)$ be a unitary vector belonging to the tangent space of $\Gamma$ defined at the point $\boldsymbol{x} \in \Gamma$. The deformation of the fiber in the $\mathbf{w}$ direction can thus be written as:

$$
\varepsilon_{1 D}=\mathbf{w}^{T} \mathbf{G} \mathbf{w}=-d_{1} \eta+\frac{d_{2}}{2} \eta^{2}+\frac{1}{2} \nabla \eta^{T} P_{\mathbf{w}} \nabla \eta,
$$

where the scalar coefficients $d_{j}$ and the projector $P_{\mathbf{w}}$ are defined as $d_{1}=\mathbf{w}^{T} \boldsymbol{B} \mathbf{w}$, $d_{2}=\mathbf{w}^{T} \boldsymbol{B} \boldsymbol{S} \mathbf{w}, P_{\mathbf{w}}=\mathbf{w} \otimes \mathbf{w}$. The constitutive stress-strain relationship is given by Eq.(30).

Let $\varrho_{\mathbf{w}}$ be the fraction of the total number of fibers aligned in the direction $\mathbf{w}$. The elastic energy of the fibers aligned in the direction $\mathrm{w}$ is of the form:

$$
\psi^{\mathbf{w}}(\eta)=\frac{1}{2} \int_{\omega} \varrho_{\mathbf{w}}\left[k_{0}+k_{1} \varepsilon_{1 D}(\eta)\right] \varepsilon_{1 D}(\eta) h^{f} \sqrt{a} d \boldsymbol{\xi}+\int_{\omega} r_{\mathbf{w}} h^{f} \sqrt{a} d \boldsymbol{\xi},
$$

where $h^{f}$ is the thickness of the smooth muscle cell layer, $r_{\mathbf{w}}$ represents the potential energy of a force acting on the fibers aligned with the direction $\mathbf{w}$.

The equilibrium equations are introduced in weak form as the scalar product with a test function of the Frechet derivative of the energy with respect to the displacement:

$$
\begin{gathered}
\Psi^{\mathbf{w}}(\eta, \chi)=\int_{\omega} \varrho_{\mathbf{w}} \nabla \chi^{T}\left[k_{0}+k_{1}\left(-d_{1}+\frac{d_{2}}{2} \eta^{2}\right)+\frac{k_{1}}{4} W\right] P_{\mathbf{w}} \nabla \eta+ \\
\varrho_{\mathbf{w}}\left[k_{0}\left(-d_{1}+d_{2} \eta\right)+k_{1}\left(-d_{1}^{2} \eta-\frac{3 d_{1} d_{2}}{2} \eta^{2}+\frac{d_{2}^{2}}{2} \eta^{3}\right)+\frac{k_{1}}{2}\left(-d_{1}+d_{2} \eta\right) W\right] \chi+ \\
\left(\delta_{\eta} r_{\mathbf{w}}\right) \chi h^{f} \sqrt{a} d \boldsymbol{\xi},
\end{gathered}
$$

where $W=\left(\nabla \eta^{T} P_{\mathbf{w}} \nabla \eta\right)$. We remark that the contribution of the first line is of membrane type, whereas the second line contains algebraic terms in the test function and hence it renders a nonlinear spring-like behavior. 
When $\eta=0$ and the SMCs are not activated, i.e. $\zeta=\bar{\zeta}$, the reference configuration is the equilibrium configuration only if the stress exerted by the fibers due to their prestress is balanced by the underlying shell. By injecting $\eta=0, \zeta=\bar{\zeta}$ into Eq.(35), we obtain:

$$
\int_{\omega}\left(-\varrho_{\mathbf{w}} \bar{k}_{0} d_{1}+\delta_{\eta} r_{\mathbf{w}}\right) \chi h^{f} \sqrt{a} d \boldsymbol{\xi}=0,
$$

hence, for any arbitrary test function $\chi$, the following holds

$$
r_{\mathbf{w}}=\varrho_{\mathbf{w}} \bar{k}_{0} d_{1} \eta
$$

where $\bar{k}_{0}=k_{0}(\bar{\zeta})$.

Remark 2. The consequence of Eq.(37) is the appearance, in the balance of the normal forces on $\Gamma$, of a force term. This is the main result of the SMCs contraction. Indeed, by combining Eq.(35) and Eq.(37) and, by setting $k_{1}=0$ for the sake of simplicity, we get:

$$
\begin{array}{r}
\Psi^{\mathbf{w}}(\eta, \chi)=\int_{\omega} \varrho_{\mathbf{w}} k_{0}(\zeta) d_{2} \eta \chi+\varrho_{\mathbf{w}} k_{0}(\zeta) \nabla \chi^{T} P_{\mathbf{w}} \nabla \eta h^{f} \sqrt{a} d \boldsymbol{\xi}+ \\
\int_{\omega} \varrho_{\mathbf{w}}\left(\bar{k}_{0}-k_{0}(\zeta)\right) d_{1} \chi h^{f} \sqrt{a} d \boldsymbol{\xi} .
\end{array}
$$

This weak formulation represents the contribution to the structure equation due to the fibers in direction $\mathbf{w}$. The effect of the activation on the wall mechanics is twofold: firstly, there is a change in the constants that characterize the passive behavior of the structure (namely in the spring-and membrane-like contributions), and secondly, a force term of the following form appears:

$$
\int_{\omega} \varrho_{\mathbf{w}}\left(\bar{k}_{0}-k_{0}(\zeta)\right) d_{1} \chi h^{f} \sqrt{a} d \boldsymbol{\xi}
$$

When $\zeta$ reaches its maximum value, this term is negative, representing a force in the normal direction that induces a negative displacement. Note that the sign of $d_{1}$ depends on the curvature along the fiber direction and it is, in general, negative when the normal is pointing outward.

Remark 3. In order to get an intuitive insight in the normal equilibrium for the structure, an example in an idealized setting is proposed. The structure is a cylinder of radius $R$, a linear Koiter shell is considered, that is in equilibrium under a pressure load. The displacement with respect to the reference configuration is constant and, hence, space and time derivatives of the displacement field vanish. Under these conditions the equilibrium displacement is the solution of an algebraic equation:

$$
\left(\frac{E h^{\kappa}}{1-\nu^{2}} c_{1}+\varrho_{\mathbf{w}} h^{f}\left(k_{0}(\zeta) d_{2}-k_{1} d_{1}^{2}\right)\right) \bar{\eta}=p-p_{i o p}-\varrho_{\mathbf{w}} h^{f} d_{1}\left(\bar{k}_{0}-k_{0}(\zeta)\right),
$$


the coefficient of $\bar{\eta}$ depends on both the mechanical properties of the structure $\left(E, \nu, \varrho_{\mathbf{w}}\right.$, $\left.k_{0}(\zeta), k_{1}\right)$ and on its geometrical properties ( $c_{1}, d_{1}, d_{2}$ which depend on the curvature). The force term depends on both the mechanical properties $\left(\varrho_{\mathbf{w}}, k_{0}(\zeta)\right)$ and the geometry $d_{1}$. Let us separate the shell contribution, the fiber contribution and the transmural presssure:

$$
\left(\frac{E h^{\kappa}}{1-\nu^{2}} c_{1}\right) \bar{\eta}=\Delta p-\sigma_{\text {fiber }},
$$

where $\sigma_{\text {fiber }}$ denotes the active and passive contributions of the fibers. When the tangential stress in a cylinder is computed by using the Koiter shell model, the following is obtained:

$$
\sigma_{\theta}=\frac{E}{1-\nu^{2}} \frac{\bar{\eta}}{R}
$$

Injecting this relationship into the equilibrium equation yields:

$$
\sigma_{\theta} \frac{h^{\kappa}}{R}=\Delta p-\sigma_{\text {fiber }} .
$$

The shell (arteriolar endothelium) is in equilibrium under the load exerted by the fibers and the transmural pressure. The tangential wall tension is simply the integral of the stress across the thickness (by making the assumption of constant stress in the section, the tension is given by $\sigma_{\theta} h^{\kappa}$ ), so that the equilibrium equation reduces to the Laplace law.

Remark 4. In general, the fibers are not parallel to only one direction. In what follows, two linearly independent unitary vectors $\mathbf{v}, \mathbf{w} \in \mathbb{T}_{\boldsymbol{x}}(\Gamma)$ and the associated fiber fractions $\varrho_{\mathbf{v}}$ and $\varrho_{\mathbf{w}}$ defined in each point of $\Gamma$ are considered. In such a case the two associated energy fields $\psi^{\mathbf{w}}$ and $\psi^{\mathbf{v}}$ sum up. If medical imaging or histological examination provide the fiber orientations, this information can be used to set $\mathbf{v}, \varrho_{\mathbf{v}}, \mathbf{w}$ and $\varrho_{\mathbf{w}}$. When this information is missing, these values can be based on a qualitative knowledge of the fibers orientation. For example, it is indicated in the study by Pournaras et al. ${ }^{28}$ p. 287 that the smooth muscle cells are oriented both circularly and longitudinally. One possible choice paralle is therefore to take the principal direction of curvature, and $\varrho_{\mathbf{v}}=\varrho_{\mathbf{w}}=\frac{1}{2}$.

With this choice, the fiber layer behaves as an isotropic homogeneous membrane.

\subsection{Summary of Section 3}

For the sake of clarity, we now summarize the model derived in the previous sections. The whole system is made of the Stokes equations

$$
\begin{cases}\left\langle\partial_{t} \boldsymbol{u}, \boldsymbol{v}\right\rangle_{\Omega}+a(\boldsymbol{u}, \boldsymbol{v})+b(p, \boldsymbol{v})=0 & \text { in } \Omega, t>0, \\ \langle\nabla \cdot \boldsymbol{u}, q\rangle_{\Omega}=0 & \text { in } \Omega, t>0, \\ \langle(\boldsymbol{I}-\boldsymbol{n} \otimes \boldsymbol{n})(\boldsymbol{u}+\eta \nabla \boldsymbol{u n}), \boldsymbol{w}\rangle_{\Gamma}=0 & \text { on } \Gamma, t>0, \\ \sigma(\boldsymbol{u}, p) \boldsymbol{n}=-p_{i n}(t) \boldsymbol{n} & \text { on } \Gamma_{i n}, t>0,\end{cases}
$$


and by the continuity of the normal velocity and of the normal stresses at the vessel wall:

$$
\begin{cases}\rho_{s} h_{s}\left\langle\partial_{t t}^{2} \eta, \chi\right\rangle_{\Gamma}+\Psi^{s}(\eta, \chi)+\left\langle p_{i o p}, \chi\right\rangle_{\Gamma}=\langle p+\eta \nabla p \cdot \boldsymbol{n}, \chi\rangle_{\Gamma} & \text { on } \Gamma, t>0 \\ \left\langle\partial_{t} \eta, \chi\right\rangle_{\Gamma}=\langle\boldsymbol{u} \cdot \boldsymbol{n}+\eta \nabla \boldsymbol{u n} \cdot \boldsymbol{n}, \chi\rangle_{\Gamma} & \text { on } \Gamma, t>0\end{cases}
$$

The behavior of the two-layer structure is modeled by $\Psi^{s}(\eta, \chi)$, defined in Equation (7).

\section{Autoregulation and pressure feedback}

As indicated in Eq.(30), the pre-stress and the elastic modulus of the SMCs are assumed to be a function of a parameter $\zeta$ describing the activation of the SMCs. Inspired by Arciero et al. ${ }^{13}$, we use the following expression for $k_{0}$ :

$$
k_{0}(\zeta)=k_{0, \text { ref }}+k_{0, a}^{\max } S(\zeta), \quad S(\zeta)=\frac{1-e^{-s\left(\zeta-p_{\text {ref }}\right)}}{1+\frac{1}{\omega} e^{-s\left(\zeta-p_{\text {ref }}\right)}},
$$

where $p_{\text {ref }}$ is a given reference pressure, $k_{0, r e f}$ is the pre-stress in the absence of activation, $\omega=-k_{0, a}^{\min } / k_{0, a}^{\max }$, where $k_{0, a}^{\max }$ and $k_{0, a}^{\min }$ are given parameters. The parameter $s$ affects the slope of the curve and can be estimated by using

$$
s=\frac{1}{p_{\max }-p_{\text {ref }}} \ln \left(\frac{1+q / \omega}{1-q}\right),
$$

where $\ln$ is the natural logarithm, and $p_{\max }$ is the value for which the active component of the pre-stress is equal to $q k_{0, a}^{\max }$. A similar behavior could be assumed for the elastic modulus of the fibers $k_{1}$, but in what follows, we simply suppose that $k_{1}=0$. A plot of the sigmoid function is presented in Figure 4 for typical values of the parameters.

We now present our strategy to compute the activation parameter $\zeta$. As mentioned in Section 3.4, SMCs react to changes in the concentration of calcium ions. The calcium ion concentration is in turn varied by several regulatory mechanisms. ${ }^{5,28,33}$ Since the focus of this work is the mechanical aspect of autoregulation, we make the simplifying assumption that the activation variable $\zeta$ directly depends on the feeding pressure. The rationale behind this choice is that an increase in the feeding pressure triggers the mechanims that will eventually affect the activation state of the smooth muscle cells. If models describing regulatory mechanisms and the concentration of calcium ions along the network were available, they could be used to provide a more physiological expression of $\zeta$.

We estimate $\zeta$ by using the mean values of the incoming pressure over the different cardiac cycles. These values are used to reconstruct $\zeta$ as a piecewise linear 


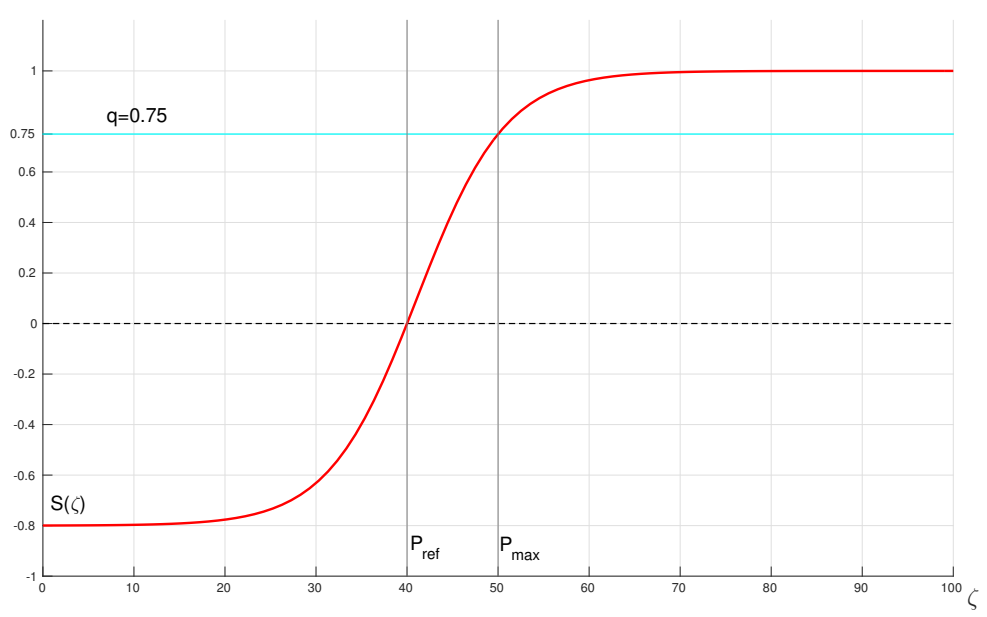

Figure 4: Sigmoid function for the parameters $\omega=0.8, P_{\max }=50, q=0.75 . S(\zeta)$ on the $y$-axis and $\zeta$ on the $x$-axis.

function in time, in the following way:

$$
\left\{\begin{array}{l}
\zeta(t)=\frac{1}{T_{i+1}-T_{i}}\left(\left(T_{i+1}-t\right) \zeta_{i}+\left(t-T_{i}\right) \zeta_{i+1}\right) \quad \forall t \in\left(T_{i}, T_{i+1}\right) \text { for } i=1,2, \ldots \\
\zeta_{i+1}=\frac{1}{\left(T_{i+1}-T_{i}\right)\left|\Sigma_{i n}\right|} \int_{T_{i}}^{T^{i+1}} \int_{\Sigma_{i n}} p d \Gamma d t, \quad \text { for } i=1,2, \ldots \\
\zeta_{0}=p_{\text {ref }}
\end{array}\right.
$$

where $\Sigma_{i n}$ denotes the inlet of the computational domain and $T_{i}$ the starting time of the $i$-th heart cycle.

As usual in computational hemodynamics, the 3D domain is truncated and the downstream vessels is taken into account by using OD Windkessel models. More precisely, each terminal vessel in our 3D network is connected to the venous pressure via an RCR compartment. For simplicity, all these compartments are assumed to share the same values for the resistances $\left(R_{\text {prox }}\right.$ and $\left.R_{\text {distal }}\right)$ and the capacitance $(C)$. The autoregulation in the Windkessel element is governed by the following hypotheses: the proximal resistance $R_{\text {prox }}$ remains constant over time; the distal resistance is given by:

$$
R_{\text {distal }}(\zeta)=R_{\text {distal,ref }}+\alpha S(\zeta) R_{\text {distal,ref }},
$$

with $\alpha=1-R_{\text {distal,max }} / R_{\text {distal,ref }}$; the capacitance varies so that the characteristic time $\tau=R_{\text {distal }}(\zeta) C(\zeta)$ remains constant. 


\section{Numerical simulations}

This section is structured as follows. First, we present a validation of the model on a test case where the mean incoming pressure coincides with the reference pressure. The values obtained for the velocity are compared with the experimental data presented in the work by Riva et al. ${ }^{39}$ Second, we present a numerical experiment where the mean incoming pressure is varied. Different flow rate-incoming pressure curves are obtained for different values of the maximum pre-stress of the fibers.

\subsection{Reference case and validation}

\subsubsection{Data}

The geometry was obtained using a retinal fundus image in the Drive dataset. ${ }^{40}$ The image was segmented and the vessels tree reconstructed by using the algorithms presented in the studies by Al-Diri et al. ${ }^{41}$ and Calivà et al. ${ }^{42}$ We considered only the inferior temporal arteriole and its branches. Twenty-five segments were obtained via the segmentation algorithm. The 3D tree was reconstructed from the 2D image by first assuming a circular section for the vessels and then by projecting the results over a sphere. The detailed bifurcations were not available from the segmentation and they were reconstructed using B-splines. The mesh generation was carried out using gmsh ${ }^{43}$ and then refined using Feflo. a, an anisotropic local remeshing sotware developed at Inria. Figure5 shows two snapshots of the geometry used for the computations. The computational mesh has 822,071 tetrahedra and 105,604 triangles on the surface, for a total number of vertices of 165,238.

In the study by Guidoboni et al. ${ }^{44}$, the authors suggest taking a pressure at the inlet of the central retinal artery that is equal to two thirds of the mean brachial arterial pressure. With typical values of systolic and diastolic brachial pressure $(120 / 80 \mathrm{mmHg})$, this gives $62 \mathrm{mmHg}$. In addition, a pressure drop of about $20 \mathrm{mmHg}$ is assumed to take place from the upstream of the central retinal artery to the downstream of the lamina cribrosa. Thus, we choose $p_{\text {ref }}=40 \mathrm{mmHg}$, which is a reference value for the pressure at the beginning of our 3D network. Regarding the outlet boundary condition we set the venous pressure at $20 \mathrm{mmHg}$, which is compatible with the value used as a reference in the work by Guidoboni et al. ${ }^{44}$ after the venules compartment. The reference values for the Windkessel parameters are $R_{\text {distal }}=6 \cdot 10^{8} \mathrm{P} \mathrm{cm}^{-3}$, $R_{\text {prox }}=6 \cdot 10^{7} \mathrm{P} \mathrm{cm}^{-3}, C=1.67 \cdot 10^{-10} \mathrm{~s} \mathrm{~cm}^{3} \mathrm{P}^{-1}$. The blood viscosity is given by $\nu^{f}=0.03 \mathrm{~cm}^{2} \mathrm{~s}^{-1}$, and its density by $\rho^{f}=1 \mathrm{~g} / \mathrm{cm}^{3}$. The structure parameters are the Young modulus of the endothelium $E=0.05 \mathrm{MPa}$ and its Poisson ratio $\nu=0.5$, the thickness of the endothelium $h^{\kappa}=5 \mu \mathrm{m}$ and the total thickness of the vessel $h^{s}=25 \mu \mathrm{m}$. The density of the structure is set equal to $1 \mathrm{~g} / \mathrm{cm}^{3}$. The fiber layer thickness is $h^{f}=20 \mu \mathrm{m}$ The mechanical properties of the fibers are the pre-stress $k_{0}=0.4 \mathrm{MPa}$ and the elastic modulus $k_{1}=0$. The intra-ocular pressure $p_{\text {iop }}$ is kept constant at $15 \mathrm{mmHg}$. 


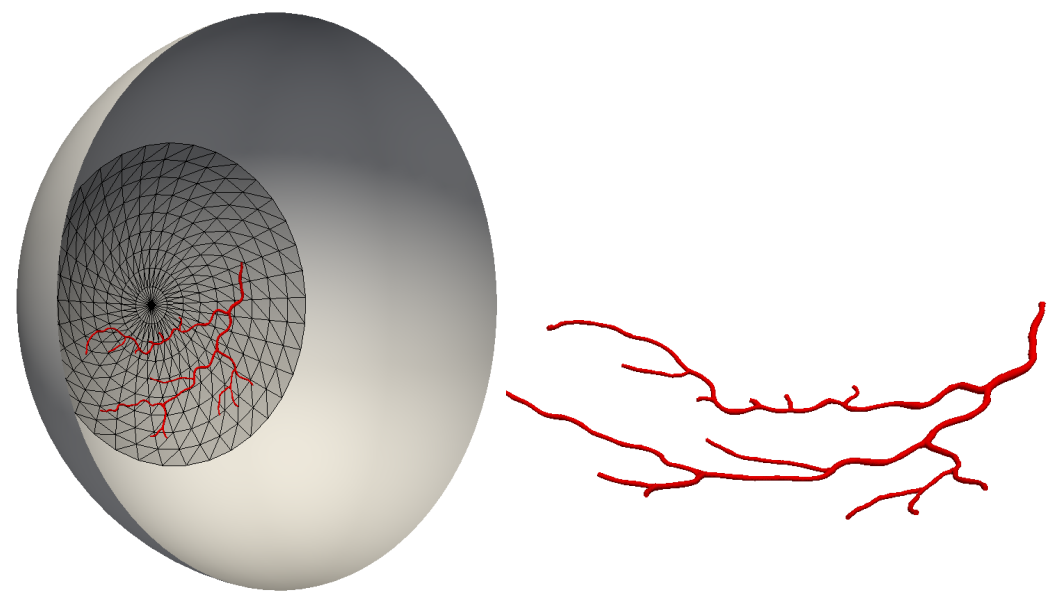

Figure 5: Computational mesh (left) and a broader view with the sphere used for the reconstruction (right).

The duration of the cardiac cycle is $0.8 s$, and the duration of diastole is $0.25 s$. The time-profile of the incoming pressure is the following:

$$
P_{\text {in }}(t)= \begin{cases}\left(p_{\text {sys }}-p_{\text {dia }}\right) \sin (\pi t / 0.25)+p_{\text {dia }} & t \in[0,0.25] \\ p_{\text {dia }} & t \in[0.25,0.8],\end{cases}
$$

where $p_{\text {dia }}=0.9 \bar{P}$ and $p_{\text {sys }}=p_{\text {dia }}+0.16 \pi \bar{P}$ are the diastolic and systolic pressure for a given value of mean pressure $\bar{P}$. A summary of these choices is presented in Table 1.

\subsubsection{Results}

In order to have a reference solution where autoregulation does not play a role and that can be used to assess the model, we set $\bar{P}=40 \mathrm{mmHg}$. We use $\boldsymbol{u}=0, \eta=0$ as initial conditions, and observe a quasi periodic behavior after two cardiac cycles. In Figure 6 we report a snapshot from the simulation taken at the time instant $t=3 \mathrm{~s}$, i.e., during the diastole. The figure also displays the surface of the computational mesh. To compare the results of our simulation with the experimental data presented in the study by Riva et al. ${ }^{39}$, we compute the mean value (in time, over a cardiac cycle) of the blood velocity at the center of different sections of the artery along the network. The value of the diameter is taken as the mean value of the diameters over the segment (between two bifurcations) which contains the section. The chosen points of the network are depicted in Figure 7, and a comparison of the data is given in Figure 8. In 
Table 1: Summary of the model parameters. In the table P stands for Poise.

\begin{tabular}{|l|l|ll|}
\hline \multicolumn{4}{|c|}{ Fluid parameters } \\
\hline$\nu^{f}$ & $0.03 \mathrm{~cm}^{2} \mathrm{~s}^{-1}$ & $\rho^{f}$ & $1 \mathrm{~g} \mathrm{~cm}^{-3}$ \\
\hline \multicolumn{3}{|c|}{ Boundary conditions and Windkessel parameters } \\
\hline$P_{\text {venous }}$ & $20 \mathrm{mmHg}$ & $P_{\text {iop }}$ & $15 \mathrm{mmHg}$ \\
$R_{\text {distal }}$ & $6 \cdot 10^{8} \mathrm{P} \mathrm{cm}^{-3}$ & $R_{\text {proximal }}$ & $6 \cdot 10^{7} \mathrm{P} \mathrm{cm}^{-3}$ \\
$C$ & $1.67 \cdot 10^{-10} \mathrm{~s} \mathrm{~cm}^{3} \mathrm{P}^{-1}$ & & \\
\hline \multicolumn{4}{|c|}{ Structure parameters } \\
\hline$\rho^{s}$ & $1 \mathrm{~g} \mathrm{~cm}^{-3}$ & $E$ & $0.05 \mathrm{MPa}$ \\
$\nu$ & 0.5 & $k_{0}$ & $0.4 \mathrm{MPa}$ \\
$k_{1}$ & 0 & $h^{k}$ & $5 \mu \mathrm{m}$ \\
$h^{f}$ & $20 \mu m$ & $h^{s}$ & $25 \mu \mathrm{m}$ \\
\hline
\end{tabular}

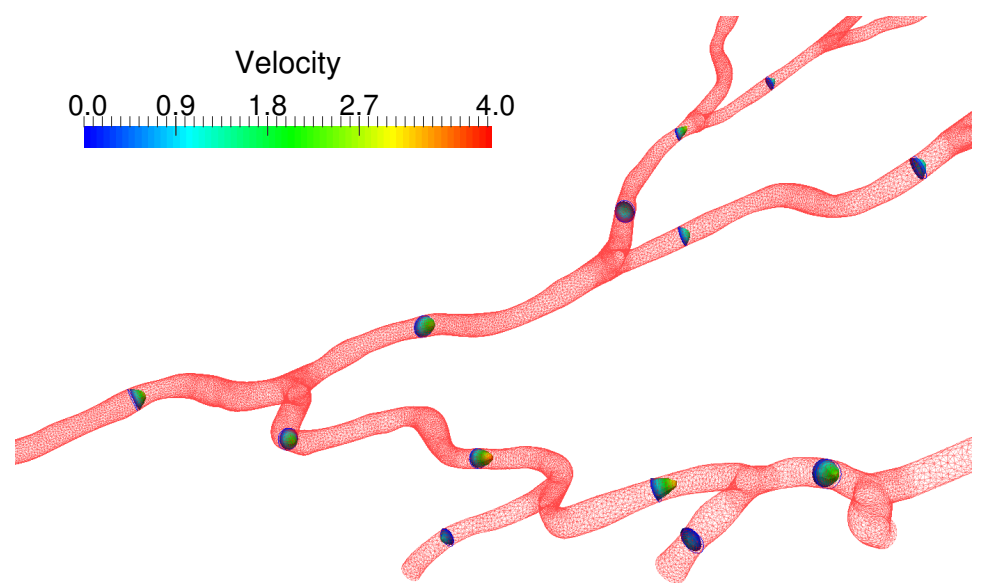

Figure 6: Velocity profiles on some slices of the domain. Values are taken during the diastolic phase of the fourth cardiac cycle $(t=3 s)$. 


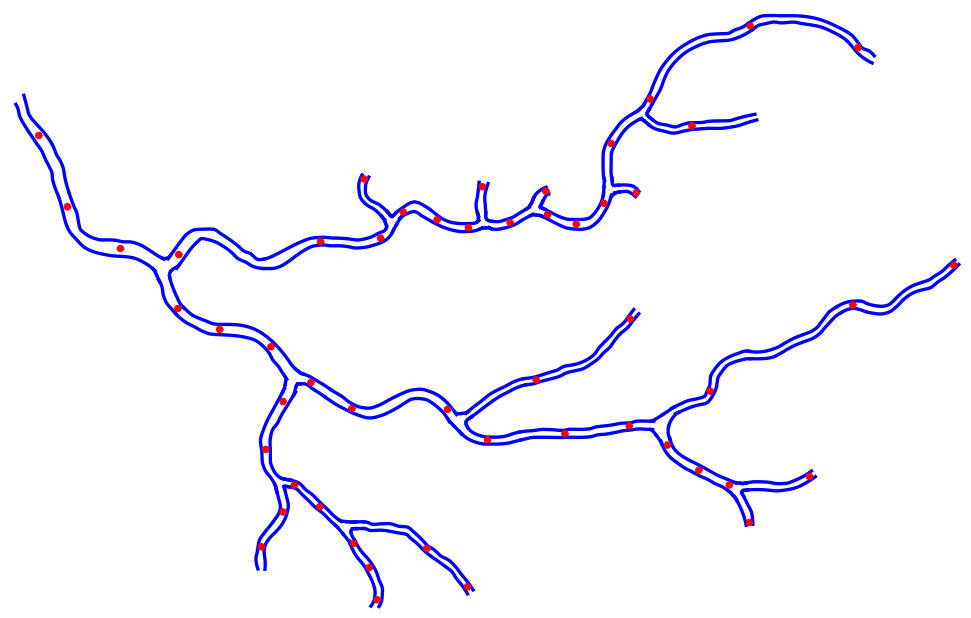

Figure 7: Points where the mean velocity has been measured for comparison with experimental data presented in Riva et al. ${ }^{39}$

order to have a fair comparison, the mean velocity over time has been computed using the same formula as was used in the study by Riva et al. $^{39}$ (one third of the systolic velocity plus two thirds of the diastolic velocity).

The results of the model are within the same range of values as the experimental data. The two sets of points also show a similar variability. However, there is a region for which either the velocity has been underestimated or the diameter has been overestimated (around $100 \mu \mathrm{m}, 1 \mathrm{~cm} / \mathrm{s}$ ). We can provide two explanations for this discrepancy. Firstly, the errors might come from the segmentation: the points with the lowest velocity are in the terminal vessels, which are the smallest and therefore the most difficult to capture with the segmentation algorithms. Secondly, it is possible that the assumption that all terminal vessels experience the same downstream equivalent resistance is too rough an approximation of reality.

\subsection{Autoregulation}

\subsubsection{Data}

For this test case we used the geometry and the data reported in Section 5.1.1. The control mechanism has been detailed in Section 4. For the present simulation, the parameters defining the sigmoid activation function (see Eq.(47)) arFe the following: $\omega=0.8$ and $P_{\max }=50 \mathrm{mmHg}, q=75 \%$. 


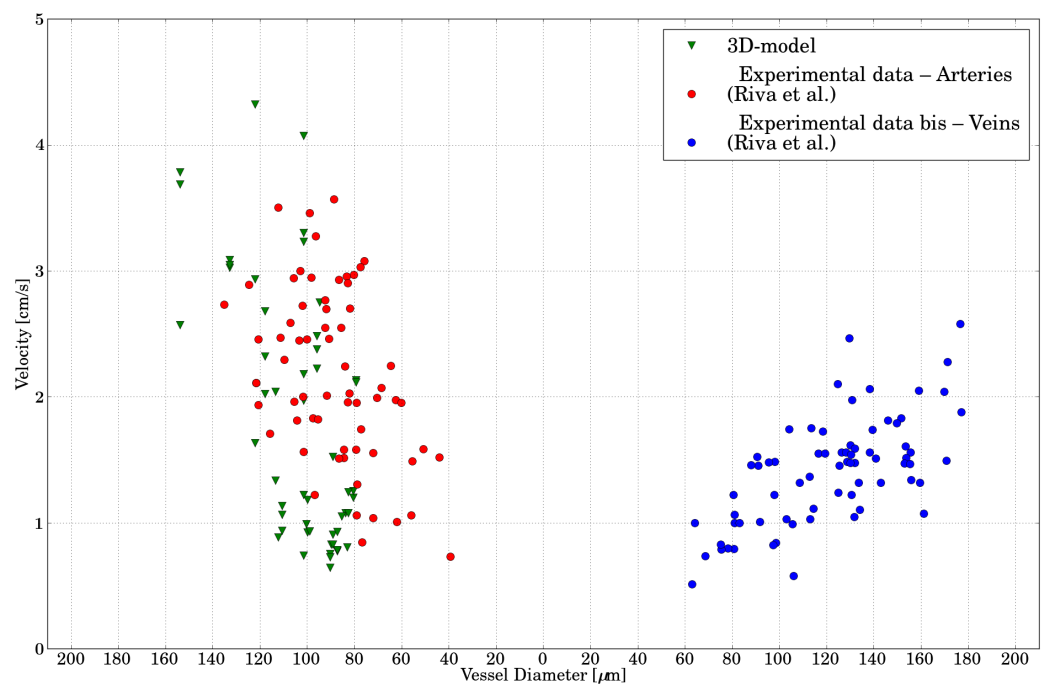

Figure 8: Comparison between the experimental data taken from Riva et al. ${ }^{39}$ Mean velocity in time ( $y$-axis) and diameter ( $x$-axis, arteries on the left side and veins on the right side). Experimental data are depicted by circles: red refers to arteries and blue refers to veins. Data computed by the proposed model are depicted by green triangles.

\subsubsection{Results}

In Figure 9 the relationship between the flow rate and the pressure at the inlet is depicted for a representative cardiac cyle in two distinct scenarios: without autoregulation (in blue) and with autoregulation (in red). Each loop is a cardiac cycle for a simulation performed by imposing a different mean incoming pressure. The chosen values are $\bar{P}=[30,40,50,60,70] \mathrm{mmHg}$. In the autoregulated case, the parameters determining the control intensity are: $k_{0, a}^{\max }=0.1 \mathrm{MPa}$ and $\alpha=0.15$. It should be noted that for a mean pressure of $\bar{P}=40 \mathrm{mmHg}$ there is only one loop since it is the reference pressure, i.e., the pressure for which the smooth muscle cells maintain their reference length. By observing these curves it can be inferred that the control mechanism acts directly on the vessels resistance. In particular, when the mean pressure is higher than the reference pressure, the flow rate is diminished, whereas when the mean pressure is lower, the SMCs action tends to increase the flow rate.

Each point of the curves represented in Figure 10 is the value of the flow entering the network for a given value of inlet pressure. The mean inlet pressure $\bar{P}$ is varied from $30 \mathrm{mmHg}$ to $70 \mathrm{mmHg}$. The parameters governing autoregulation are chosen as follows: $k_{0, a}^{\max }$ takes three values: 0 (no autoregulation), $0.05 \mathrm{MPa}$ and $0.1 \mathrm{Mpa}$. The autoregulation parameter $\alpha$, defined in equation (49), indicates how much the 


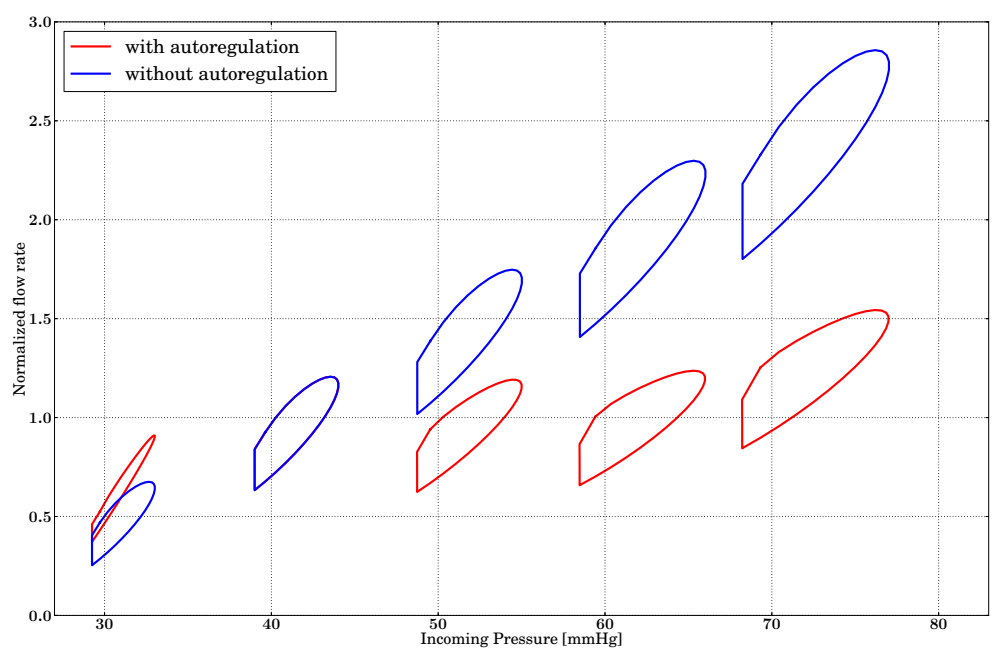

Figure 9: Normalized flow rate ( $y$-axis) with respect to incoming pressure ( $x$-axis). Each circle represents one cardiac cycle from a different simulation. The blue circles correspond to the simulations without autoregulation: $K_{0, a}^{\max }=0$ and $\alpha=0$, for different values of $\bar{P}$. The red circles refer to the autoregulation parameters $K_{0, a}^{\max }=0.1 \mathrm{MPa}$ and $\alpha=0.15$.

downstream circulation is able to vary its overall resistance. Two different cases were considered: $\alpha=0$ (no autoregulation) and $\alpha=0.15$ (for which the maximum value of distal resistance is equal $1.15 R_{\text {distal, ref }}$ ). For each numerical experiment four cardiac cycles are simulated. The values of the flow are taken in the last cycle and the flow is normalized with respect to the value obtained for $\bar{P}=p_{\text {ref }}=40 \mathrm{mmHg}$. Figure 10 shows that using $k_{0, a}^{\max }=0.1 \mathrm{MPa}$ and using autoregulation in the Windkessel model (circles, green), it is possible to replicate a plateau in the flow rate-pressure relationship. This result is similar to that obtained in the study by Arciero et al. ${ }^{13}$ with a OD approach. The impact of autoregulation in the Windkessel for this choice of parameters can be observed by comparing the green curve (circles) with the red one (down triangles), which was obtained with the same $k_{a, 0}^{\max }$ and by turning the Windkessel autoregulation off $(\alpha=0)$.

\section{Limitations and conclusion}

In this work, we have proposed a first attempt at modeling autoregulation in a 3D network of retinal arteries. Our approach is based on a simplified fluid-structure model whose computational cost is of the same order as the cost of a pure fluid problem. The 


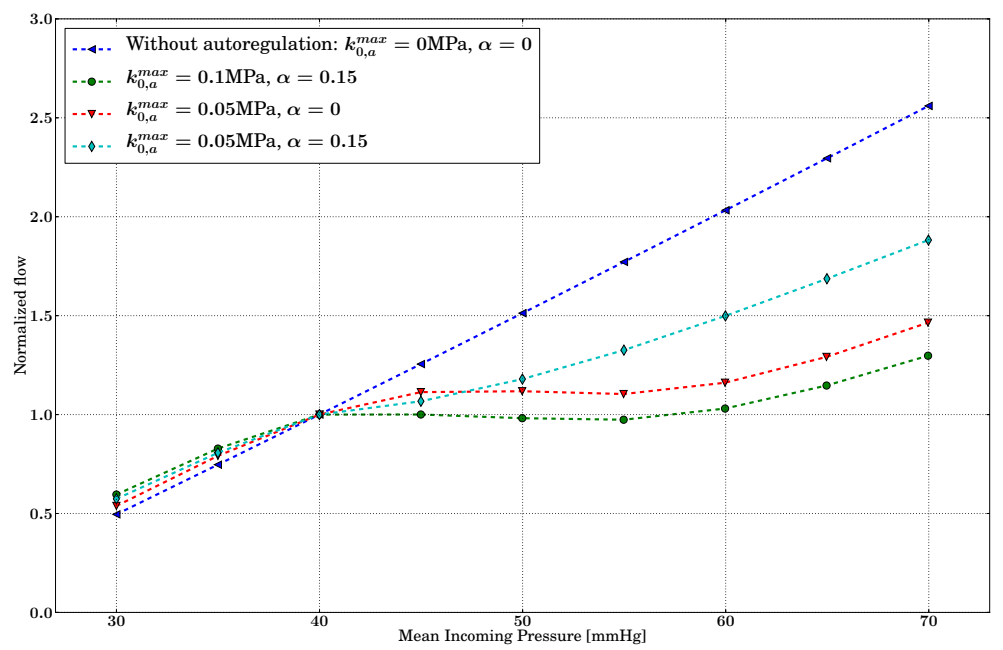

Figure 10: Autoregulation curves. Mean incoming pressure on the fourth cardiac cycles ( $x$-axis) and normalized flow rate over the fourth cardiac cycle ( $y$-axis). Different lines correspond to different values for the autoregulation parameters. Each point on a curve refers to a different simulation with a given mean incoming pressure.

model used for the wall includes smooth muscle fibers, whose active constitutive law has been derived by approximating physiological models proposed in the literature. The simulations performed in a real network of 25 segments of retinal arteries have provided velocities which are in good agreement with published experimental data. By varying the parameters of the active component of the constitutive law, we have been able to reproduce flow rate-pressure curves which are comparable with experimental data or results obtained with OD models. In particular, a characteristic plateau of the flow rate has been found for pressures ranging from 40 to $60 \mathrm{mmHg}$.

To the best of our knowledge, this study is the first to propose 3D simulations of blood flow in a real network of retinal arteries, including an autoregulation mechanism. It can be viewed as a first step toward a more complete 3D model of the hemodynamic of the eye. In spite of encouraging results, many limitations remain and the model could be improved in various ways.

Firstly, the diameter of the vessels considered in this work is below $200 \mu \mathrm{m}$, which means that the hemodynamics is in a microcirculation regime. ${ }^{27,28}$ In such vessels, the Fahraeus effect, the Fahraeus-Lindqvist effect and plasma skimming may be relevant in determining the distribution of hematocrit and the velocity profile. ${ }^{45,46}$

Secondly, our autoregulation model describes how smooth muscle fibers control 
the blood flow, but not the physiological mechanisms that trigger the contraction or the relaxation. The feedback mechanism could be improved to include other aspects than only the inlet pressure.

Besides these limitations, future works could also improve the models used for the downstream vasculature, and should address other important phenomena like the interaction with other compartments, such as the lamina cribrosa or the intraocular pressure.

\section{Acknowledgements}

The authors would like to thank Bashir Al-Diri, Andrew Hunter and Francesco Calivà for the geometry of the arterial network, and the anonymous referees for their comments and suggestions.

This research was made possible by a Marie Curie grant from the European Commission in the framework of the REVAMMAD ITN (Initial Training Research network), Project number 316990.

\section{References}

1. Schubert R, Mulvany MJ. The myogenic response: established facts and attractive hypotheses.. Clin Sci (Lond) 1999;96(4):313-326. Available from: http://toxnet.nlm.nih.gov/cgi-bin/sis/search2/ r?dbs+hsdb:@term+@rn+7440-70-2 PubMed PMID: 10087237.

2. Kohner EM, Patel V, Rassam SM. Role of blood flow and impaired autoregulation in the pathogenesis of diabetic retinopathy.. Diabetes 1995;44(6):603-607. Available from: http://toxnet.nlm.nih. gov/cgi-bin/sis/search2/r?dbs+hsdb:@term+@rn+7782-44-7 PubMed PMID: 7789621. doi: 10.2337/ diab.44.6.603.

3. Rassam SM, Patel V, Kohner EM. The effect of experimental hypertension on retinal vascular autoregulation in humans: a mechanism for the progression of diabetic retinopathy.. Exp Physiol 1995;80(1):53-68. Available from: http://onlinelibrary.wiley.com/resolve/openurl?genre=article\&sid=nlm:pubmed\&issn=0958-0670\&date=1995\&volume=80\&issue=1\&spage=53 PubMed PMID: 7734138.

4. Grunwald JE, Riva CE, Stone RA, Keates EU, Petrig BL. Retinal autoregulation in open-angle glaucoma.. Ophthalmology 1984;91(12):1690-1694. Available from: http://www.diseaseinfosearch. org/result/3065 PubMed PMID: 6521997.

5. Jeppesen P, Aalkjær C, Bek T. Myogenic response in isolated porcine retinal arterioles, Current eye research 27. Ophthalmology 2003;91(12):217-222. Available from: http://www.diseaseinfosearch. org/result/3065 PubMed PMID: 6521997.

6. Jeppesen P, Sanye-Hajari J, Bek T. Increased blood pressure induces a diameter response of retinal arterioles that increases with decreasing arteriolar diameter.. Invest Ophthalmol Vis Sci 2007;48(1):328-331. Available from: https://www.researchgate.net/publication/e/pm/17197550?ln_ $\mathrm{t}=\mathrm{p} \& \ln \_\mathrm{o}=$ linkout PubMed PMID: 17197550. doi: 10.1167/iovs.06-0360.

7. Blum M, Bachmann K, Wintzer D, Riemer T, Vilser W, Strobel J. Noninvasive measurement of the Bayliss effect in retinal autoregulation.. Graefes Arch Clin Exp Ophthalmol 1999;237(4):296-300. 
Available from: http://ClinicalTrials.gov/search/term=10208262\%20\%5BPUBMED-IDS\%5D PubMed PMID: 10208262.

8. Harris A, Arend O, Bohnke K, Kroepfl E, Danis R, Martin B. Retinal blood flow during dynamic exercise.. Graefes Arch Clin Exp Ophthalmol 1996;234(7):440-444. Available from: https://www.nlm.nih.gov/ medlineplus/exerciseandphysicalfitness.html PubMed PMID: 8817287.

9. Dumskyj MJ, Eriksen JE, Dor' e CJ, Kohner EM. Autoregulation in the human retinal circulation: assessment using isometric exercise, laser doppler velocimetry, and computer-assisted image analysis. Microvascular research 1996;51(3):378-392. Available from: https://www.researchgate.net/publication/e/pm/8992235?ln_t=p\&ln_o=linkout PubMed PMID: 8992235. doi: 10.1006/mvre.1996.0034.

10. Robinson F, Riva CE, Grunwald JE, Petrig BL, Sinclair SH. Retinal blood flow autoregulation in response to an acute increase in blood pressure.. Invest Ophthalmol Vis Sci 1986;27(5):722-726. Available from: https://www.researchgate.net/publication/e/pm/3700021?ln_t=p\&ln_o=linkout PubMed PMID: 3700021.

11. Nagaoka T, Mori F, Yoshida A. Retinal artery response to acute systemic blood pressure increase during cold pressor test in humans.. Invest Ophthalmol Vis Sci 2002;43(6):1941-1945. Available from: https://www.nlm.nih.gov/medlineplus/highbloodpressure.html PubMed PMID: 12037003.

12. Arciero JC, Carlson BE, Secomb TW. Theoretical model of metabolic blood flow regulation: roles of ATP release by red blood cells and conducted responses. American Journal of Physiology - Heart and Circulatory Physiology 2008;295(4). Available from: http://ajpheart.physiology.org/cgi/pmidlookup?view=long\&pmid=18689501 PubMed PMID: 18689501. doi: 10.1152/ajpheart.00261.2008.

13. Arciero J, Harris A, Siesky B, Amireskandari A, Gershuny V, Pickrell A, et al. Theoretical analysis of vascular regulatory mechanisms contributing to retinal blood flow autoregulation.. Invest Ophthalmol Vis Sci 2013 Aug;54(8):5584-5593. Available from: http://europepmc.org/abstract/MED/23847315 PubMed PMID: 23847315. doi: 10.1167/iovs.12-11543.

14. Hester RL, Hammer LW. Venular-arteriolar communication in the regulation of blood flow.. Am J Physiol Regul Integr Comp Physiol 2002;282(5). Available from: http://ajpregu.physiology.org/cgi/ pmidlookup?view=long\&pmid=11959667 PubMed PMID: 11959667. doi: 10.1152/ajpregu.00744.2001.

15. Moireau P, Bertoglio C, Xiao N, Figueroa CA, Taylor C, Chapelle D, et al. Sequential identification of boundary support parameters in a fluid-structure vascular model using patient image data. Biomechanics and Modeling in Mechanobiology 2012;12(3):475-496. Available from: http://link.springer. com/10.1007/s10237-012-0418-3 doi: 10.1007/s10237-012-0418-3.

16. Bertoglio C, Barber D, Gaddum N, Valverde I, Rutten M, Beerbaum P, et al. Identification of artery wall stiffness: In vitro validation and in vivo results of a data assimilation procedure applied to a 3D fluidstructure interaction model. Journal of Biomechanics 2014;47(5):1027-1034. Available from: http:// linkinghub.elsevier.com/retrieve/pii/S0021929014000098 PubMed PMID: 24529756. doi: 10.1016/j. jbiomech.2013.12.029.

17. Fernández M, Gerbeau JF. Algorithms for fluid-structure interaction problems, Cardiovascular Mathematics. Modeling and simulation of the circulatory system. Springer Verlag, 2009, Ch. 9; .

18. Bazilevs Y, Calo V, Zhang Y, Hughes TJ. Isogeometric fluid- structure interaction analysis with applications to arterial blood flow. Computational Mechanics 2006;38(4-5):4-5. Available from: http://link. springer.com/10.1007/s00466-006-0084-3 doi: 10.1007/s00466-006-0084-3.

19. Crosetto P, Reymond P, Deparis S, Kontaxakis D, Stergiopulos N, Quarteroni A. Fluid-structure interaction simulation of aortic blood flow. Computers \& Fluids 2011;43(1):46-57. Available from: http:// linkinghub.elsevier.com/retrieve/pii/S0045793010003452 doi: 10.1016/j.compfluid.2010.11.032.

20. Moireau P, Xiao N, Astorino M, Figueroa CA, Chapelle D, Taylor CA, et al. External tissue support and fluid-structure simulation in blood flows.. Biomech Model Mechanobiol 2011 Feb;11(1-2):1-18. Available from: https://www.researchgate.net/publication/e/pm/21308393?ln_t=p\&ln_o=linkout PubMed PMID: 21308393. doi: 10.1007/s10237-011-0289-z.

21. Fernández MA, Landajuela M, Vidrascu M. Fully decoupled time-marching schemes for incompressible fluid/thin-walled structure interaction. Journal of Computational Physics 2015;297:156-181. 
Available from: http://linkinghub.elsevier.com/retrieve/pii/S0021999115003289 doi: 10.1016/j. jcp.2015.05.009.

22. Figueroa CA, Vignon-Clementel IE, Jansen KE, Hughes TJR, Taylor CA. A coupled momentum method for modeling blood flow in three-dimensional deformable arteries. COMPUTER METHODS IN APPLIED MECHANICS AND ENGINEERING 2006 Aug;195(41-43):5685-5706. Available from: http:// linkinghub.elsevier.com/retrieve/pii/S004578250500513X doi: 10.1016/j.cma.2005.11.011.

23. Nobile F, Vergara $C$. An effective fluid-structure interaction formulation for vascular dynamics by generalized robin conditions. SIAM Journal on Scientific Computing 2008;30(2):731-763. Available from: http://epubs.siam.org/doi/abs/10.1137/060678439 doi: 10.1137/060678439.

24. Pironneau O. Numerical Simulations of Coupled Problems in Engineering, Vol. 33 of Computational Methods in Applied Sciences. Springer International Publishing; 2014.

25. Colciago CM, Deparis S, Quarteroni A. Comparisons between reduced order models and full 3D models for fluid-structure interaction problems in haemodynamics. Journal of Computational and Applied Mathematics 2014;265:120-138. Available from: http://linkinghub.elsevier.com/retrieve/pii/ S0377042713005049 doi: 10.1016/j.cam.2013.09.049.

26. M. Aletti, J.-F. Gerbeau, D. Lombardi, A simplified fluid-structure model for arterial flow. Application to retinal hemodynamics., submitted (2015).

27. Wong TY, Klein R, Klein BEK, Meuer SM, Hubbard LD. Retinal vessel diameters and their associations with age and blood pressure.. Invest Ophthalmol Vis Sci 2003;44(11):4644-4650. Available from: http://www.scholaruniverse.com/ncbi-linkout?id=14578380 PubMed PMID: 14578380.

28. Pournaras CJ, Rungger-Brändle E, Riva CE, Hardarson SH, Stefansson E. Progress in retinal and eye research. 2008.

29. Ciarlet PG, Vol, e.V. (Mathematical) . of Studies in Mathematics and its Applications. Amsterdam: North-Holland Publishing Co; 2000.

30. Murtada S, Kroon M, Holzapfel GA. A calcium-driven mechanochemical model for prediction of force generation in smooth muscle.. Biomech Model Mechanobiol 2010 Mar;9(6):749-762. Available from: http://toxnet.nlm.nih.gov/cgi-bin/sis/search2/r?dbs+hsdb:@term+@rn+7440-70-2 PubMed PMID: 20354752. doi: 10.1007/s10237-010-0211-0.

31. Milnor WR. Cardiovascular physiology. Oxford University Press; 1990.

32. Laties AM. Central retinal artery innervation. Absence of adrenergic innervation to the intraocular branches.. Arch Ophthalmol 1967;77(3):405-409. Available from: http://archopht.jamanetwork.com/ article.aspx?doi=10.1001/archopht.1967.00980020407021 PubMed PMID: 4960032. doi: 10.1001/ archopht.1967.00980020407021.

33. Bek T. Regional morphology and pathophysiology of retinal vascular disease.. Prog Retin Eye Res 2013 Jul;36:247-259. Available from: https://www.nlm.nih.gov/medlineplus/retinaldisorders.html PubMed PMID: 23892140. doi: 10.1016/j.preteyeres.2013.07.002.

34. Hai CM, Murphy RA. Cross-bridge phosphorylation and regulation of latch state in smooth muscle. Am J Physiol 254 (1 Pt 1988;1:99-106.

35. Yang J, Clark JW, Bryan RM, Robertson C. The myogenic response in isolated rat cerebrovascular arteries: smooth muscle cell model. Medical Engineering \& Physics 2003;25(8):691-709. Available from: http://linkinghub.elsevier.com/retrieve/pii/S1350453303001000 doi: 10.1016/S13504533(03)00100-0.

36. Yang J, Clark JW, Bryan RM, Robertson CS. The myogenic response in isolated rat cerebrovascular arteries: vessel model. Medical Engineering \& Physics 2003;25(8):711-717. Available from: http:// linkinghub.elsevier.com/retrieve/pii/S1350453303001012 PubMed PMID: 12900185. doi: 10.1016/ S1350-4533(03)00101-2.

37. Stålhand J, Klarbring A, Holzapfel GA. Smooth muscle contraction: mechanochemical formulation for homogeneous finite strains.. Prog Biophys Mol Biol 2007 Aug;96(1-3):465-481. Available from: http://www.scholaruniverse.com/ncbi-linkout?id=17884150 PubMed PMID: 17884150. doi: 10.1016/j.pbiomolbio.2007.07.025. 
38. Sharifimajd B, Stålhand J. A continuum model for excitation-contraction of smooth muscle under finite deformations. Journal of Theoretical Biology 2014;355:1-9. Available from: http://linkinghub.elsevier.com/retrieve/pii/S0022519314001519 PubMed PMID: 24657629. doi: 10.1016/j. jtbi.2014.03.016.

39. Riva CE, Grunwald JE, Sinclair SH, Petrig BL. Blood velocity and volumetric flow rate in human retinal vessels.. Invest Ophthalmol Vis Sci 1985;26(8):1124-1132. Available from: https://www.researchgate. net/publication/e/pm/4019103?ln_t=p\&ln_o=linkout PubMed PMID: 4019103.

40. Staal J, Abràmoff MD, Niemeijer M, Viergever MA, van Ginneken B. Ridge-Based Vessel Segmentation in Color Images of the Retina. IEEE Trans. Med. Imaging 2004;23(4):501-509. Available from: http:// ieeexplore.ieee.org/lpdocs/epic03/wrapper.htm?arnumber=1282003 PubMed PMID: 15084075. doi: 10.1109/TMI.2004.825627.

41. Al-Diri B, Hunter A, Steel D. An active contour model for segmenting and measuring retinal vessels. IEEE Transactions on Medical Imaging 2009;28(9):1488-1497. Available from: http://www.scholaruniverse.com/ncbi-linkout?id=19336294 PubMed PMID: 19336294. doi: 10.1109/TMI.2009.2017941.

42. F. Calivà, M. Aletti, B. Al-Diri, A. Hunter, A new tool to connect blood vessels in fundus retinal images, in: Proceedings of 37th Annual international conference of the IEEE engineering in medicine and biology society, 2015.

43. Geuzaine C, Remacle JF. Gmsh: A 3-D finite element mesh generator with built-in pre-and post-processing facilities. International Journal for Numerical Methods in Engineering 2009;79(11):13091331. Available from: http://doi.wiley.com/10.1002/nme.2579 doi: 10.1002/nme.2579.

44. Guidoboni G, Harris A, Cassani S, Arciero J, Siesky B, Amireskandari A, et al. Intraocular pressure, blood pressure, and retinal blood flow autoregulation: a mathematical model to clarify their relationship and clinical relevance.. Invest Ophthalmol Vis Sci 2014 May;55(7):4105-4118. Available from: http://europepmc.org/abstract/MED/24876284 PubMed PMID: 24876284. doi: 10.1167/iovs.1313611.

45. Pries A, Neuhaus D, Gaehtgens P, Blood . viscosity in tube flow: dependence on diameter and hematocrit. American Journal of Physiology 1992;263:1770-1770.

46. Pries AR, Ley K, Claassen M, Gaehtgens P. Red cell distribution at microvascular bifurcations.. Microvasc Res 1989;38(1):81-101. Available from: https://www.researchgate.net/publication/e/ pm/2761434?ln_t=p\&ln_o=linkout PubMed PMID: 2761434. 